\title{
MAPEAMENTO DE USO E COBERTURA DA TERRA E ANÁLISE DA ESTRUTURA DA PAISAGEM NA BACIA DO RIO URAIM
}

\author{
Lucimar Costa Pereira \\ manas, Belém, PA, Brasil \\ lu.costa0708@gmail.com
}

Arlete Silva de Almeida Programa de Pós-Graduação em Geografia. Coordenadoria de Ciências da Terra e Ecologia - CCTE.

Belém/PA, Brasil arlete@museu-goldi.br

Bruno Ferreira Monteiro Museu Paraense Emílio Goeldi. Campus de Pesquisa, Belém, PA, Brasil brunoferreiramont@gmail.com

Wanja Janayna de Miranda Lameira Professora. Secretaria Municipal de Educação - SEMEC PA wjlameira@hotmail.com

Sabrina Pereira de Assunção

Universidade do Estado do Pará. Centro de Ciências Naturais e Tecnologia. Belém, PA, Brasil sabrina.e.ambiental@gmail.com

\section{RESUMO}

A pesquisa objetiva mapear e analisar o uso e cobertura da terra e a estrutura da paisagem florestal, a partir de fragmentos florestais (manchas) e métricas de paisagem na bacia do rio Uraim, Paragominas-PA. A classificação de uso e cobertura foi realizada no software ENVI v. $5.3 \mathrm{com}$ aplicação do algoritmo de Máxima verossimilhança (MAXVER). Já os fragmentos florestais foram analisados a partir de métricas de paisagem, no software ArcMap v. 10.5. A classificação indicou que, as classes com maior predomínio na área da bacia do Uraim eram floresta primária (27,50\%), vegetação secundária (21,46\%) e agricultura (17,68\%). O mapeamento das áreas florestadas contabilizou 29.504 fragmentos na bacia. A maior parte dos remanescentes florestais da bacia do Uraim são compostos por fragmentos muito pequenos. Os fragmentos maiores apresentaram menor efeito de borda em relação aos menores, entretanto, as métricas de forma indicaram irregularidade mais acentuada para os fragmentos com maior área. Nesse contexto, vale ressaltar que o perfil de desenvolvimento de atividades agropecuárias na bacia do Uraim é o fator chave para a fragmentação florestal e a análise desses fragmentos contribui como auxílio na tomada de decisões para melhor gestão da bacia, considerando a necessidade de corredores ecológicos.

Palavras-chave: Classes. Métricas. Fragmentos.

\section{LAND USE AND LAND COVER MAPPING AND ANALYSIS OF THE LANDSCAPE STRUCTURE IN THE URAIM RIVER BASIN}

\begin{abstract}
The research aims to map and analyze land use and land cover and forest landscape structure from forest fragments (patches) and landscape metrics in the Uraim river basin, Paragominas-PA. The classification of use and coverage was performed using ENVI v. 5.3 with application of the Maximum Likelihood algorithm (MAXVER). Forest fragments were analyzed using landscape metrics with ArcMap v. 10.5 software. The classification indicated that the most predominant classes in the Uraim basin area were primary forest $(27.50 \%)$, secondary vegetation $(21.46 \%)$ and agriculture (17.68\%). Mapping of forested areas accounted for 29,504 fragments in the basin. Mapping of forest fragments has shown that most of the remnants of the Uraim basin are composed of very small fragments. Most of the forest remnants of the Uraim basin are composed of very small fragments. Larger fragments had lower edge effect
\end{abstract}


than smaller fragments, however, the shape metrics indicated more irregularity for the larger fragments. In this context, it is noteworthy that the development profile of agricultural activities in the Uraim basin is the key factor for forest fragmentation and the analysis of these fragments contributes as an aid in decision making for better management of the basin, considering the need for ecological corridors.

Keywords: Classes. Metrics. Fragments.

\section{INTRODUÇÃO}

As bacias hidrográficas são caracterizadas como um conjunto ligado por cursos d'água cujo escoamento superficial possui apenas um exutório. O percentual de água que a bacia recebe depende de alguns fatores, entre eles a extensão que a mesma ocupa, a taxa de infiltração de água no solo, a precipitação, evaporação e escoamento, entre outros, ligados de maneira direta ao ciclo da água (ALVES, et al., 2019).

Pela Política Nacional de Recursos Hídricos-PNRH, a bacia hidrográfica é adotada como uma unidade de estudo, planejamento, gerenciamento dos recursos hídricos, de desenvolvimento econômico e social. É nesta unidade que se aplicam os instrumentos da PNRH (BRASIL, 1997).

Esta unidade de estudo permite a integração de dados interdisciplinares para um melhor gerenciamento dos recursos da área, e uma das ferramentas utilizadas para essa finalidade é o geoprocessamento (PEREIRA et al., 2019). A confiabilidade e a rapidez no processo de aquisição e processamento de dados proporcionados pelas geotecnologias trazem maior facilidade de se mapear o uso e a cobertura de grandes áreas (NASCIMENTO e FERNANDES, 2017; RODRIGUES et al., 2019).

Diante da crescente demanda por recursos naturais, para suprimento de necessidades básicas ou para desenvolvimento de atividades econômicas (pequena e grande escala), as bacias hidrográficas sofrem impactos ambientais diretos e indiretos, em virtude da forma desordenada de ocupação e uso descontrolado dos recursos naturais (NASCIMENTO e FERNANDES, 2017). Entre os impactos ambientais consideráveis destacam-se, processo de erosão, impermeabilização do solo, suscetibilidade a inundações, assoreamento, entre outros.

Nessa vertente, frente ao desenvolvimento intenso de atividades econômicas no município de Paragominas-PA, em áreas localizadas no limite geográfico da bacia do Uraim, esta pesquisa considerou como problemática principal o seguinte questionamento: Qual a dinâmica de uso e ocupação da terra na bacia do Uraim e qual o grau de fragmentação da estrutura da paisagem florestal na bacia?

Para entendimento da problemática em questão, tornam-se importantes estudos que possam subsidiar a gestão adequada e a análise da dinâmica socioambiental nas bacias hidrográficas. De tal forma, o mapeamento dos distintos tipos de uso e análise de fragmentação florestal são pontos imprescindíveis para a compreensão da dinâmica vivenciada na bacia. A análise da distribuição de classes de tamanho de fragmentos é útil para a definição de estratégias para a conservação da biodiversidade em áreas protegidas (VIANA e PINHEIRO, 1998: REIS e ALVES, 2017).

Os levantamentos realizados dos tipos de usos e controle do processo de fragmentação florestal são ações requisitadas para contenção dos efeitos de borda, da perda da biodiversidade e baixo fluxo gênico. Além desses fatores, é necessário considerar que os processos ecológicos, tais como dispersão de sementes e polinização, que dependem de determinados animais, sofrem alterações em função da fragmentação (RIBOLDI et al., 2017).

Diante do exposto, a pesquisa objetiva mapear e analisar de maneira quantiqualitativa o uso e cobertura da terra e a estrutura da paisagem florestal, a partir de fragmentos florestais (manchas) e métricas de paisagem na bacia do rio Uraim, Paragominas-PA. 


\section{MATERIAIS E MÉTODOS}

\section{Localização e caracterização da área de estudo}

A área de estudo consiste na bacia do rio Uraim, com área de $5.110,83 \mathrm{~km}^{2}$. A mesma fica localizada em dois municípios da região nordeste do Estado do Pará, com maior parte $(86,99 \%)$ no município de Paragominas e uma parte menor $(12,47 \%)$ no município de Nova Esperança do Piriá. A bacia é cortada pela BR-010 e tem como curso d'água principal o Rio Uraim que serve como fonte de abastecimento do município de Paragominas. Dentro da área da bacia existem diversas estradas de chão que não possuem nomes definidos pelo Departamento Nacional de Infraestrutura de Transportes - DNIT. Apenas a BR-010 e as PAs 125 e 256 possuem nomenclatura definida (Figura 1).

Figura 1 - Localização da bacia do rio Uraim.

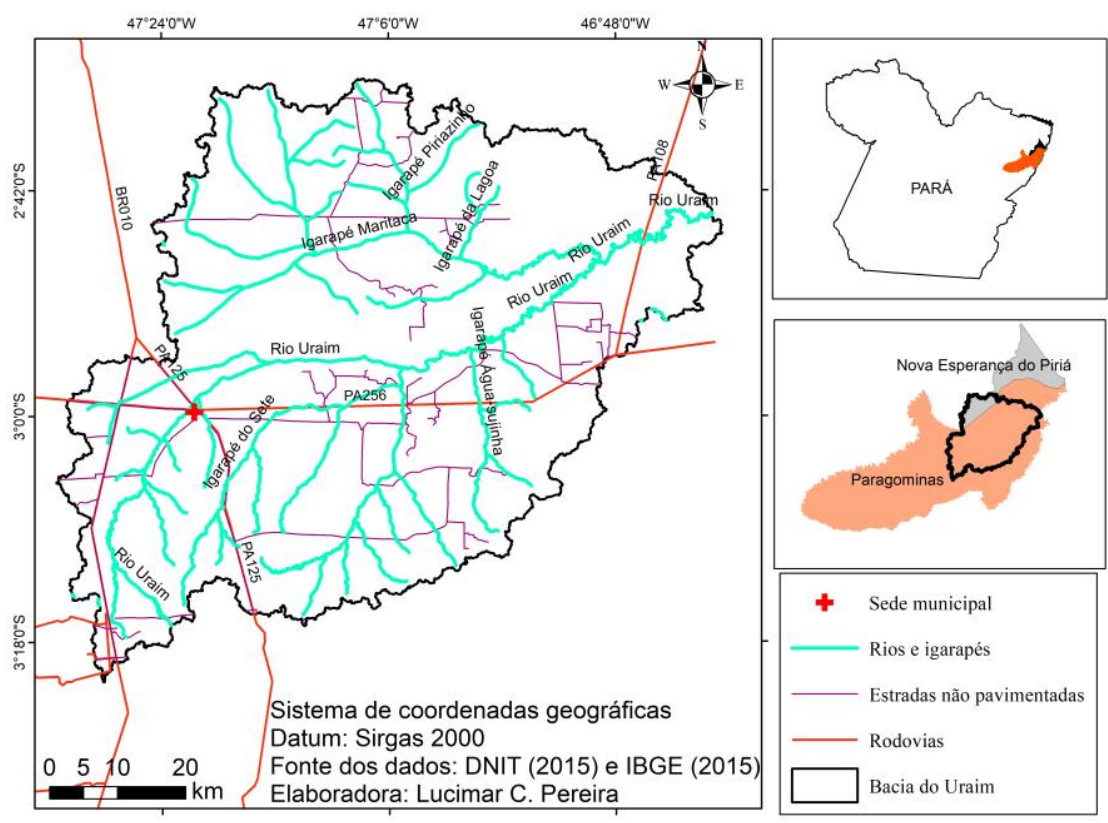

Organizadores: Autores (2019).

O solo predominante na bacia é do tipo Latossolo amarelo (EMBRAPA, 2019), mesmo tipo que compõe grande parte do município de Paragominas. O clima é do tipo quente e úmido, característico da região Paragominense.

\section{Delimitação da bacia do rio Uraim}

Anteriormente ao processo de classificação de uso e cobertura da terra, foi feita delimitação geográfica da bacia do Uraim, com o intuito de obter-se um produto com mais precisão, considerando a resolução espacial e radiométrica do Modelo Digital de Elevação-MDE usado. Utilizou-se MDE, do sensor Palsar, satélite Alos, disponível na plataforma da National Aeronautics and Space Administration - NASA (Administração Nacional da Aeronáutica e Espaço), datado de abril de 2011. O MDE obtido apresenta resolução espacial e radiométrica de $12,5 \mathrm{~m}$ e 16 bits, respectivamente (NASA, 2011).

O limite da bacia do Uraim foi extraído, de maneia automática, a partir da ferramenta hydrology, no ambiente do software ArcMap 10.5, no laboratório de sensoriamento remoto do Centro de Ciências da Terra, Campus de Pesquisa do Museu Paraense Emílio Goeldi, Belém-PA, o qual 
detém de licença para utilização do referido software. O método utilizado na extração subdividiu-se em quatro etapas, sendo as mesmas: preenchimento de depressões ("fill"), direção de fluxo ("flow direction"), fluxo acumulado ("flow accumulation") e delimitação de bacias ("Watershed").

\section{Classificação de uso e cobertura}

Para classificação do uso e cobertura da terra foi utilizada imagem de 02/07/2018, do sensor OLI, satélite Landsat 8, disponível no site do Serviço Geológico dos Estados Unidos - USGS (2018). A imagem compreende à órbita/ponto 222/62, com resolução espacial e radiométrica de $30 \mathrm{~m}$ e 8 bits, respectivamente.

A classificação supervisionada ocorreu com aplicação do algoritmo de Máxima verossimilhança (MAXVER) no software Envi v. 5.3, no laboratório de Sensoriamento Remoto do Centro de Ciências da Terra, nas dependências do campus de Pesquisa do Museu Paraense Emilio Goeldi, Belém-PA, detentor da licença adequada do software citado. Foram definidas 10 classes, sendo as mesmas, floresta primária, floresta degradada, vegetação secundária, agricultura, pastagem, reflorestamento, solo exposto, água, nuvem e sombra. A classificação foi validada a partir de confirmação de pontos duvidosos em trabalho de campo e avaliada a partir de partir da matriz de confusão, por meio do índice kappa, de acordo com Landis e Koch (1977). Os autores associam valores de kappa à qualidade da classificação de acordo com a escala apresentada na tabela 1.

Tabela 1 - Qualidade da classificação supervisionada associada aos valores de índice kappa.

\begin{tabular}{cc} 
Índice kappa & Qualidade \\
\hline$<0.00$ & Péssima \\
\hline $0.00-0.20$ & Ruim \\
\hline $0.21-0.40$ & Razoável \\
\hline $0.41-0.60$ & Boa \\
\hline $0.61-0.80$ & Muito boa \\
\hline $0.81-1.00$ & Excelente \\
\hline
\end{tabular}

Fonte: Landis e Koch (1977 p.165).

O índice kappa é um dos parâmetros mais utilizados de maneira eficiente para quantificação da exatidão de levantamentos de uso da terra. O valor do índice é obtido a partir da adoção de uma referência para comparar-se com os produtos dos mapeamentos realizados. Nesse viés, a análise é feita por meio de matrizes de confusão ou matrizes de erro, o que gera posteriormente o índice kappa (COHEN, 1960).

O produto da classificação foi exportado em formato de arquivo vetorial (shapefile) e importado no software Arcmap v. 10.5, devidamente licenciado, conforme especificado anteriormente. No referido software foi realizado o cálculo da área $\left(\mathrm{em}^{2} \mathrm{~km}^{2}\right)$ correspondente a cada classe definida.

\section{Mapeamento dos fragmentos florestais}

Para análise de fragmentos (manchas) por meio de métricas de paisagem foi utilizada a classe floresta primária, obtida no processo de classificação supervisionada. A partir do arquivo de fragmentação florestal, foi quantificada a área de cada fragmento usando-se a tabela de 
atributos do próprio arquivo vetorial poligonal, o que tornou possível comparar os tamanhos das diversas manchas florestais encontradas na área da bacia. $O$ tamanho dos fragmentos foi classificado da seguinte forma: muito pequeno ( $<5 \mathrm{ha}$ ), pequeno (5-10 ha), médio (10-100 ha) e grande (>100 ha) (JUVANHOL et al., 2011).

\section{Aplicação de métricas de paisagem para os fragmentos florestais}

A estrutura da paisagem florestal foi analisada a partir de métricas de paisagem. As métricas para os fragmentos florestais foram obtidas no ambiente do software ArcMap v. 10.5, a partir da extensão Patch Analyst (analisador de manchas), no laboratório de Sensoriamento Remoto do Centro de Ciências da Terra, nas dependências do campus de Pesquisa do Museu Paraense Emilio Goeldi, Belém-PA. A análise dos fragmentos foi baseada em método proposto por Pirovani (2010). Foram utilizados os seguintes grupos de métricas: área; borda e forma dos fragmentos (manchas).

Para uma melhor comparação entre o grau de conservação e o tamanho dos fragmentos florestais mapeados, foi realizada a análise das métricas de paisagem para cada uma das classes de tamanho (muito pequena, pequena, média e grande) e também para os fragmentos como um todo, a fim de se obter valores médios dos grupos de métricas para todos os fragmentos da bacia.

O detalhamento das métricas utilizadas nesta pesquisa, juntamente com suas siglas e significados pode ser visualizado no quadro 1.

Quadro 1 - Índices de ecologia da paisagem gerados em nível de classe a partir do Patch Analyst junto aos fragmentos florestais.

\begin{tabular}{|c|c|c|c|c|}
\hline Grupo & Sigla & Métrica & Unidade & Observação \\
\hline \multirow{4}{*}{ 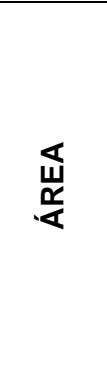 } & $\mathrm{CA}$ & Área da classe & Hectare (ha) & $\begin{array}{c}\text { Somatório das áreas de todas as manchas ou } \\
\text { fragmentos florestais presentes na área em } \\
\text { estudo. }\end{array}$ \\
\hline & MPS & $\begin{array}{l}\text { Tamanho médio dos } \\
\text { fragmentos ( } \\
\text { manchas) }\end{array}$ & Hectare (ha) & $\begin{array}{l}\text { Soma do tamanho das manchas dividido pelo } \\
\text { número de manchas }\end{array}$ \\
\hline & NUMP & $\begin{array}{l}\text { Número de } \\
\text { manchas }\end{array}$ & Adimensional & Número total de manchas paisagem/classe. \\
\hline & PSSD & $\begin{array}{l}\text { Desvio padrão do } \\
\text { tamanho da mancha }\end{array}$ & Hectare (ha) & $\begin{array}{l}\text { Razão da variância do tamanho } \\
\text { das manchas. }\end{array}$ \\
\hline \multirow{2}{*}{ 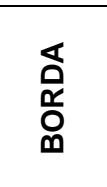 } & TE & Total de bordas & Metro (m) & $\begin{array}{l}\text { Extremidade total de todas as } \\
\text { manchas. É a soma de perímetro } \\
\text { de todas as manchas. }\end{array}$ \\
\hline & ED & Densidade de borda & $\mathrm{m} / \mathrm{ha}$ & $\begin{array}{l}\text { Quantidade de extremidades relativa à área } \\
\text { da paisagem. }\end{array}$ \\
\hline \multirow{2}{*}{ 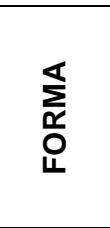 } & MSI & $\begin{array}{l}\text { Índice de forma } \\
\text { médio }\end{array}$ & Adimensional & $\begin{array}{l}\text { É igual a um quando todas as manchas forem } \\
\text { circulares e aumenta com a crescente } \\
\text { irregularidade da forma da mancha. }\end{array}$ \\
\hline & AWMSI & $\begin{array}{l}\text { Índice de forma } \\
\text { de área média } \\
\text { ponderada }\end{array}$ & Adimensional & $\begin{array}{l}\text { Difere do MSI, porque manchas } \\
\text { maiores terão mais peso que as menores. }\end{array}$ \\
\hline
\end{tabular}

Fonte: MCGARIGAL e MARKS (1995).

\section{RESULTADOS E DISCUSSÃO}

A delimitação automática da bacia do Uraim indicou uma área de $5.110,83 \mathrm{~km}^{2}$ e perímetro com 414,57 km. Já a acurácia da classificação de uso e cobertura da terra, avaliada a partir do índice kappa (LANDIS e Koch, 1977, p.165), indicou qualidade excelente com índice igual a 0,82 . 


\section{Uso e cobertura do solo}

A quantificação de uso e cobertura da terra indicou que em 2018 , de $5.110,83 \mathrm{~km}^{2}$ de área da bacia hidrográfica, $27,50 \%$ eram floresta primária, $21,46 \%$ correspondiam a vegetação secundária e 17,68\% correspondiam a áreas de agricultura. Com quantitativo mais reduzido que estas classes, entretanto não menos importante, as áreas de floresta degradada contabilizaram $9,89 \%$, a classe pastagem representou $9,50 \%$, e solo exposto $4,92 \%$. A cobertura de nuvens e sombra apresentou percentual de 6,93\%. As classes de água, reflorestamento e área urbana apresentaram os percentuais mais reduzidos, com 1,27\%, $0,57 \%$ e $0,27 \%$, respectivamente (Tabela 2 ).

Tabela 2 - Quantitativo de classes de uso e cobertura da terra na bacia do Uraim, ParagominasPA.

\begin{tabular}{c|c|c}
\hline Classes & Área $\left.\mathbf{( k m}^{\mathbf{2}}\right)$ & Percentual (\%) \\
\hline Floresta primária & 1405,57 & 27,50 \\
\hline Vegetação secundária & 1096,65 & 21,46 \\
\hline Agricultura & 903,85 & 17,68 \\
\hline Floresta degradada & 505,46 & 9,89 \\
\hline Pastagem & 485,62 & 9,50 \\
\hline Solo exposto & 251,24 & 4,92 \\
\hline Sombra & 250,45 & 4,90 \\
\hline Nuvem & 103,51 & 2,03 \\
\hline Água & 65,13 & 1,27 \\
\hline Reflorestamento & 29,37 & 0,57 \\
\hline Área urbana & 13,98 & 0,27
\end{tabular}

Organizadores: Autores (2019).

Apesar da classe de floresta primária apresentar-se com valor mais elevado que os outros tipos de uso é necessário considerar o percentual de área de floresta degradada, que representa quase $10 \%$ da área da bacia. Além disso, as extensas áreas de agricultura, capoeira e pastagem revelam que o grau de conservação das florestas está comprometido pelo intenso desenvolvimento de tais atividades. Tal fato é verificado ao se analisar o número de imóveis rurais presentes na área da bacia, que demonstram que as áreas de floresta primária se constituem essencialmente em áreas de reserva legal de tais imóveis rurais (Figuras 3 A e 3B).

Figura 3 - Comparativo da disposição das áreas de floresta primária (A) com áreas de reserva legal (B) na bacia do Uraim.
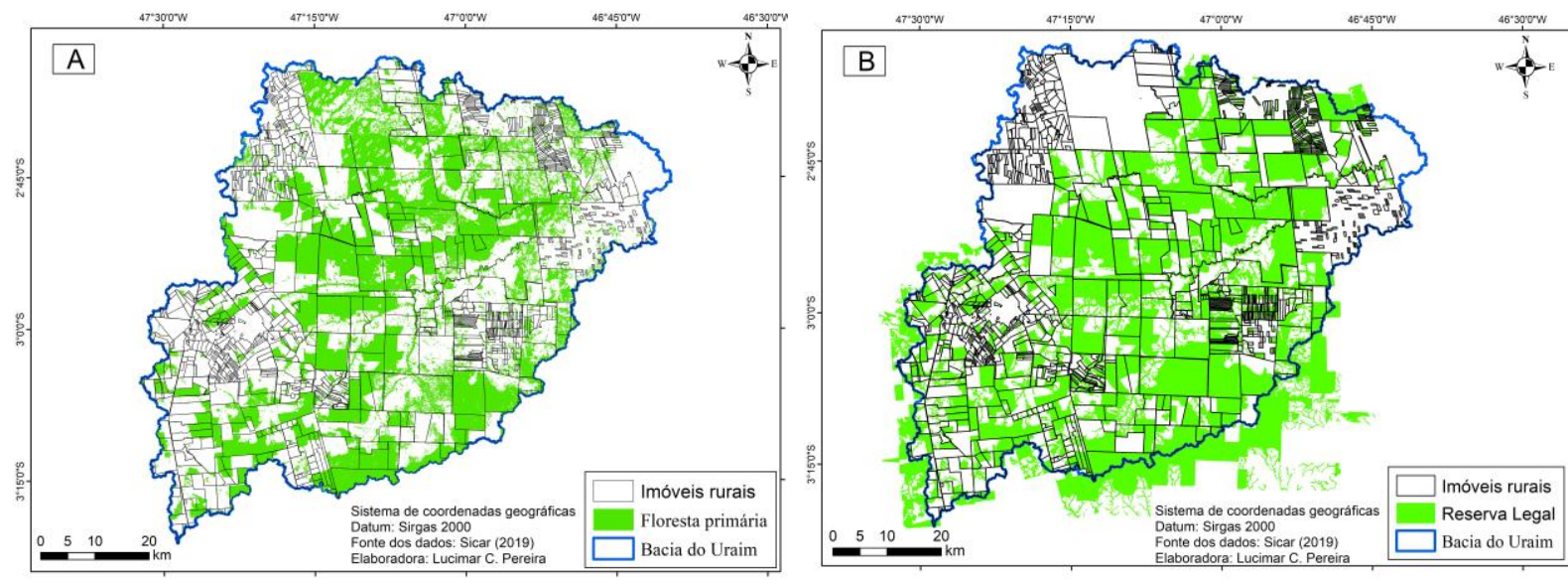

Organizadores: Autores (2019) 
Essa vegetação, em forma de reservas das propriedades rurais, representa um avanço na busca por recuperação das áreas desmatadas, iniciada a partir da implementação do Projeto Municípios Verdes em Paragominas, no ano de 2009. Entretanto, o número elevado de imóveis rurais implica em menor quantidade de vegetação com vistas somente à conservação da biodiversidade na área da bacia. As fontes desse fato estão na caracterização histórica de Paragominas como um município de desenvolvimento de atividades exploradoras de recursos naturais, inicialmente com agricultura desenvolvida por colonos, passando pela pecuária e exploração madeireira. Por volta de 1994 e 1995, ocorre a instalação da produção de grãos em grande escala e a monocultura de árvores, o que passa a demandar abertura de extensas áreas (IMAZON, 2013), ação "necessária" para desenvolvimento de todas as atividades econômicas de grande porte em Paragominas (FERNANDES, 2011).

Nesse contexto, a agropecuária se destaca, onde o desenvolvimento desse setor dentro da bacia do Uraim representa $27,18 \%$ da mesma, o que praticamente se iguala ao quantitativo de floresta primária $(27,50 \%)$. Já as áreas de vegetação secundária também se destacam pelo percentual próximo $(21,46 \%)$ aos de floresta primária e de agropecuária. Essas áreas de vegetação secundária são encontradas em diferentes estágios de desenvolvimento e muitas vezes estão associadas ao sistema agrossilvipastoril utilizadas após um período de pousio (PIROVANI, 2010).

Em pesquisa realizada por Nascimento e Fernandes (2017) na bacia hidrográfica do Igarapé da Prata em Capitão Poço-PA, os autores também identificaram elevado percentual de áreas de agricultáveis, pastagem e vegetação secundária. Os autores concluíram que a dinâmica das classes de pastagem e vegetação secundária demonstra a formação de um ciclo de uso e ocupação da terra na bacia, pelo qual as atividades desenvolvidas caracterizam o solo como pasto e a posterior inatividade deste propiciará a regeneração da vegetação na área e, que posterirormente se caracterizará como vegetação secundária e poderá voltar a ser pasto ou não.

Tais resultados indicam que o desenvolvimento de atividades agropecuárias em bacias hidrográficas contribui gravemente para a conversão elevada de áreas florestadas em outros tipos de uso da terra, em pouco espaço de tempo. O processo inadequado de ocupação por pastagens e culturas, principalmente com pouca cobertura vegetal nas áreas de preservação permanente dentro da área da bacia hidrográfica provoca sério desiquilíbrio ambiental (RODRIGUES et al., 2019).

No tocante à classe reflorestamento, em pesquisa desenvolvida por Santos et al. (2017), sobre o programa Municípios Verdes em Paragominas, os autores concluíram que o reflorestamento não atingiu as expectativas do programa, pois, somente em 7 de 22 registros se observou características de vegetação em estágio de recuperação, o que pode ser um indício da existência de um processo de reflorestamento, porém de maneira incipiente.

A área urbana do município de Paragominas, presente dentro da bacia, ainda não alcançou proporções com significância elevada, capaz de ser considerada uma grande problemática causadora de impactos ambientais na bacia do Uraim. As alterações, provocadas pelo avanço de ocupação urbana, que merecem mais atenção, podem ser refletidas nas áreas de APP presentes no perímetro urbano, como verificado em pesquisa realizada por Pereira et al. (2016). Os autores identificaram uma quantidade elevada de vegetação rasteira (13,37 ha), fruto de ocupações nas margens do rio Uraim ao longo do perímetro urbano. O solo exposto oriundo do processo de ocupação das áreas de APP pode acarretar problemáticas ambientais como assoreamento, erosão e inundações, sendo este último evento de ocorrência constante em Paragominas.

No que se refere à distribuição das classes ao longo da área de estudo, os fragmentos de floresta primária se encontram nas regiões sul, noroeste, sudeste e na área central da bacia. A classe de reflorestamento está presente principalmente na área central da bacia, em pequena quantidade, quando comparada às demais classes de uso. Áreas de solo exposto e vegetação secundária estão distribuídas em diversas partes da unidade de pesquisa, principalmente em proximidades de cursos d'água. Já as classes de agricultura e pastagem foram verificadas nas 
Lucimar Costa Pereira

Arlete Silva de Almeida

Mapeamento de uso e cobertura da terra e análise da

Bruno Ferreira Monteiro

estrutura da paisagem na bacia do rio Uraim

Wanja Janayna de Miranda Lameira

Sabrina Pereira de Assunção

proximidades da área urbana e nas regiões sul e noroeste da bacia. A área urbana fica localizada na região sul-sudoeste da bacia (Figura 4).

Figura 4 - Mapa de uso e cobertura da terra na bacia do Uraim, Paragominas-PA.

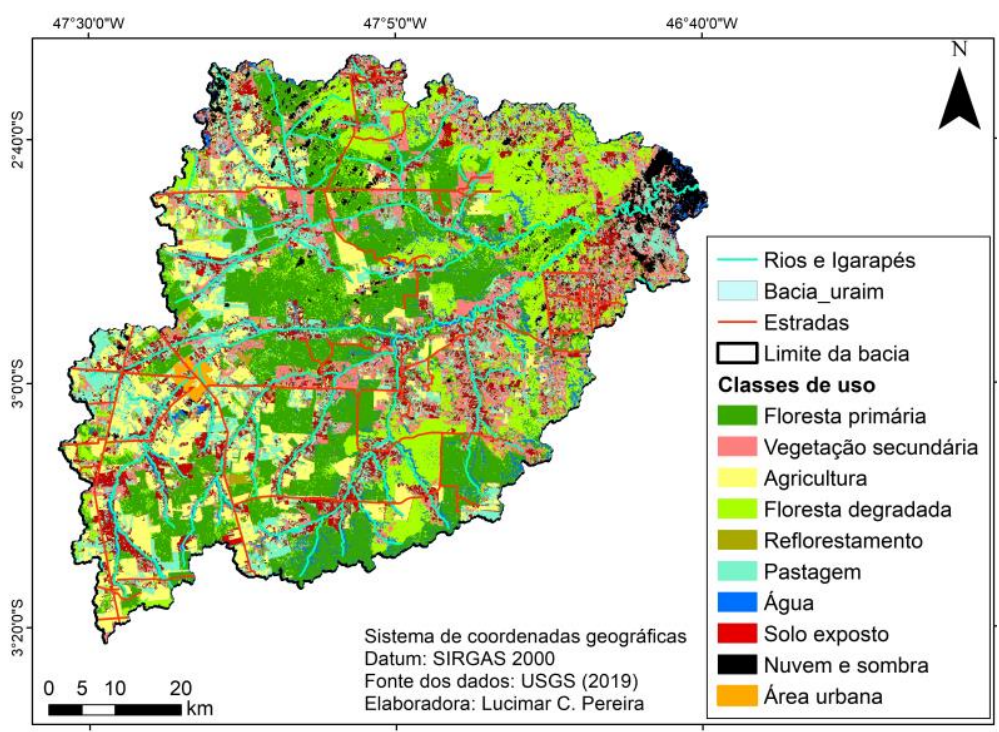

Organizadores: Autores (2019)

Pela análise do mapa de uso da terra é possível verificar que as áreas de preservação permanente dos cursos d'água na bacia estão comprometidas devido à presença, principalmente, de áreas de capoeira e solo exposto (Figuras 5A e 5B), além de pastagem em alguns trechos, o que pode propiciar processos erosivos com consequente poluição do corpo hídrico.

Figura 5 - Representação das classes capoeira e solo exposto na bacia do Uraim nas proximidades do curso d’água denominado Igarapé do Sete.

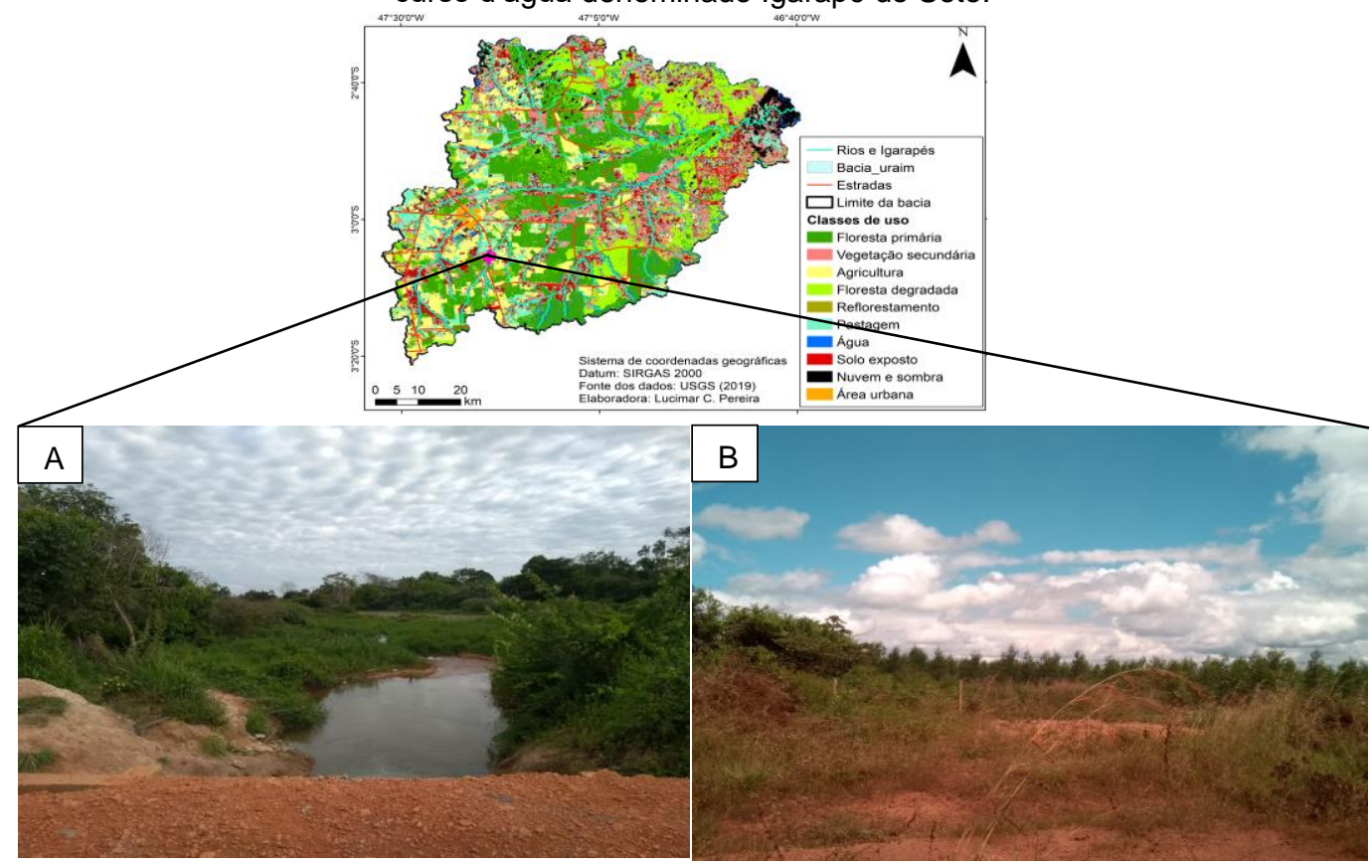

Fonte: Autores (2019). 
Alves et al. (2019), em pesquisa realizada na bacia do Ribeirão Douradinho, no sudoeste de Goiás, concluíram que pelo fato da bacia apresentar predominância de área agrícola, o desenvolvimento das atividades agropecuárias observadas nas áreas de preservação permanente compromete o estado de conservação do recurso hídrico.

São destinadas na bacia do Uraim extensas áreas para o desenvolvimento de atividades agropecuárias, como foi verificado no processo de classificação, as quais localizam-se sobretudo, nas proximidades de estradas, "escondidas" atrás de um extenso corredor de vegetação nas margens das estradas. Essa vegetação está presente em vários estágios sucessionais.

As áreas de reflorestamento são constituídas principalmente de espécies exóticas, com fins lucrativos, sendo esta prática desenvolvida em áreas paralelas à área de floresta primária (Figura 6). A produção de madeira de reflorestamento de paricá de empresas e produtores na microrregião de Paragominas, formada pelos municípios de Paragominas, Ulianópolis, Dom Eliseu e Rondon do Pará é destinada exclusivamente para a manufatura de compensado (SANTOS et al., 2018).

Figura 6 - Classe reflorestamento na área da bacia, ao lado da classe de floresta primária na região sul da bacia.

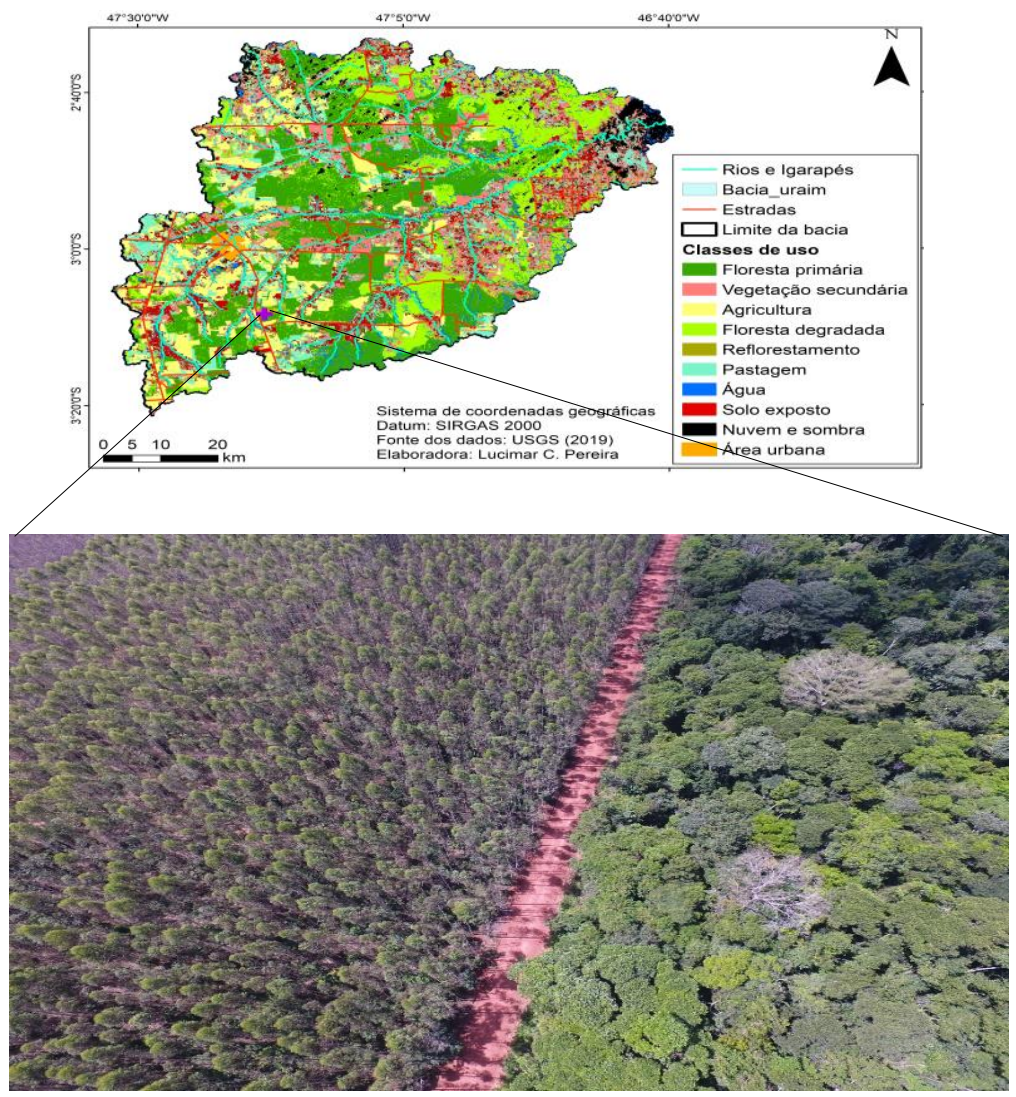

Fonte: Autores (2019).

\section{Fragmentos florestais}

O mapeamento das áreas florestadas contabilizou 29.504 fragmentos florestais (manhas) em toda bacia. Este número corresponde a uma área de 140. 557 ha de remanescentes florestais, o que equivale a $27,50 \%$ da bacia. Essa quantidade de áreas florestais demonstra um estado de conservação alterado, impulsionado pela forte caracterização da área como local de desenvolvimento de atividades econômicas. 
O mapeamento dos fragmentos florestais demonstrou que a maior parte dos remanescentes da bacia do Uraim são compostos por fragmentos muito pequenos $(<5 \mathrm{ha})$, correspondendo a $94,96 \%$ (28.016) do total de fragmentos florestais encontrados na área de estudo. A predominância de fragmentos muito pequenos na unidade de estudo implica em consequências negativas para manutenção da biodiversidade, haja vista que a riqueza de espécies diminui à medida que os fragmentos sofrem redução de tamanho. Nesse viés as espécies raras, com pequena área de distribuição, assim como aquelas que necessitam de habitats muito amplos ou especializados, são as mais suscetíveis aos efeitos da fragmentação (SAUNDERS et al., 1991: TURNER, 1996).

Rex et al. (2018), em pesquisa realizada na bacia do rio Pequeno-PR também constataram que a cobertura florestal da bacia está fortemente fragmentada, com um número elevado de fragmentos menores que 10 ha. Os autores concluíram que a fragmentação ocorreu como consequência da quantidade elevada de áreas de campo.

A classe de fragmentos pequenos (entre 5 e $10 \mathrm{ha}$ ) representou um percentual de $1,11 \%$ (328) do número total de 29.504 fragmentos, e a classe dos médios (entre 10 e 100 ha) representou $0,87 \%$ (256) do total de fragmentos florestais mapeados. A classe dos fragmentos grandes, composta por aqueles acima de 100 ha, obteve um valor baixo em relação ao número total (65), o que equivale a $0,22 \%$.

Riboldi et al. (2017), em pesquisa realizada no município de Salgado Filho-PR, também observaram um número reduzido de fragmentos maiores e concluíram que este fato pode refletir diretamente na perda da biodiversidade local em função dos efeitos de borda.

Apesar dos fragmentos grandes estarem em menor número, a área correspondente a estes, representa $85,99 \%$ (120.484,63 ha) da área total de fragmentos florestais. Os fragmentos muito pequenos $(10.177,26 \mathrm{ha})$ e pequenos $(2.242,19 \mathrm{ha})$, em relação à área total, representaram $8,86 \%$ enquanto os médios $(7.215,24 \mathrm{ha}$ ) representaram $5,15 \%$ (Figura 7 ).

Figura 7. Frequência absoluta e relativa das áreas de fragmentos florestais.

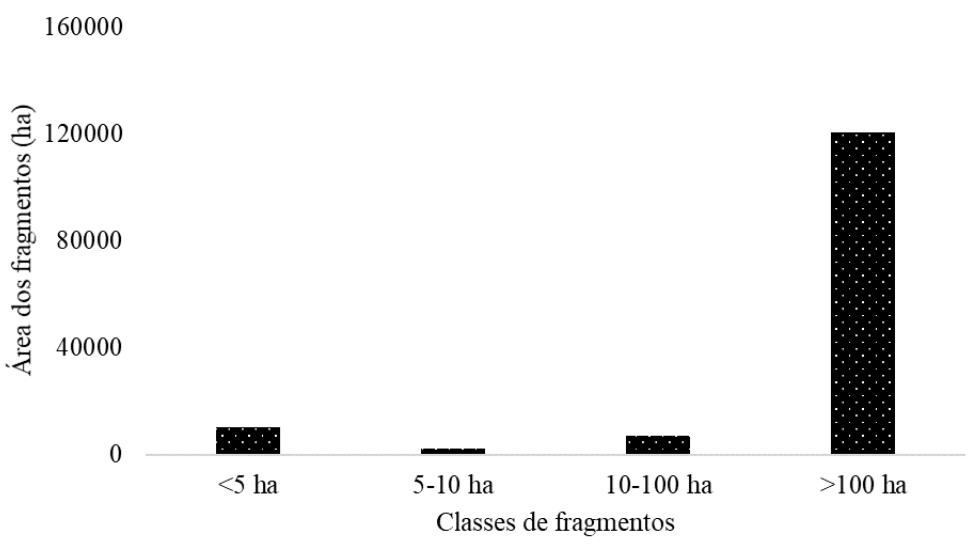

Organizadores: Autores (2019).

A relação entre o número de fragmentos e da área que estes ocupam, em muitos casos se caracteriza de maneira inversa. Isto significa que os grandes fragmentos possuem menor percentual em número, entretanto, representam uma parcela maior da área total dos remanescentes florestais na área da bacia. Os fragmentos pequenos possuem maior número de unidades, porém, a soma das áreas destes representa menor percentual da área total dos fragmentos florestais mapeados (JUVANHOL et al., 2011). Os fragmentos florestais que mais se destacam espacialmente são os maiores que 100 ha e os menores que 5 ha, sendo a classe dos maiores distribuídas por quase toda a bacia enquanto os menores se concentram principalmente nas regiões sudeste e noroeste (Figura 10). 
Figura 10 - Distribuição das classes de tamanho dos fragmentos florestais na bacia do Uraim, Paragominas-PA.

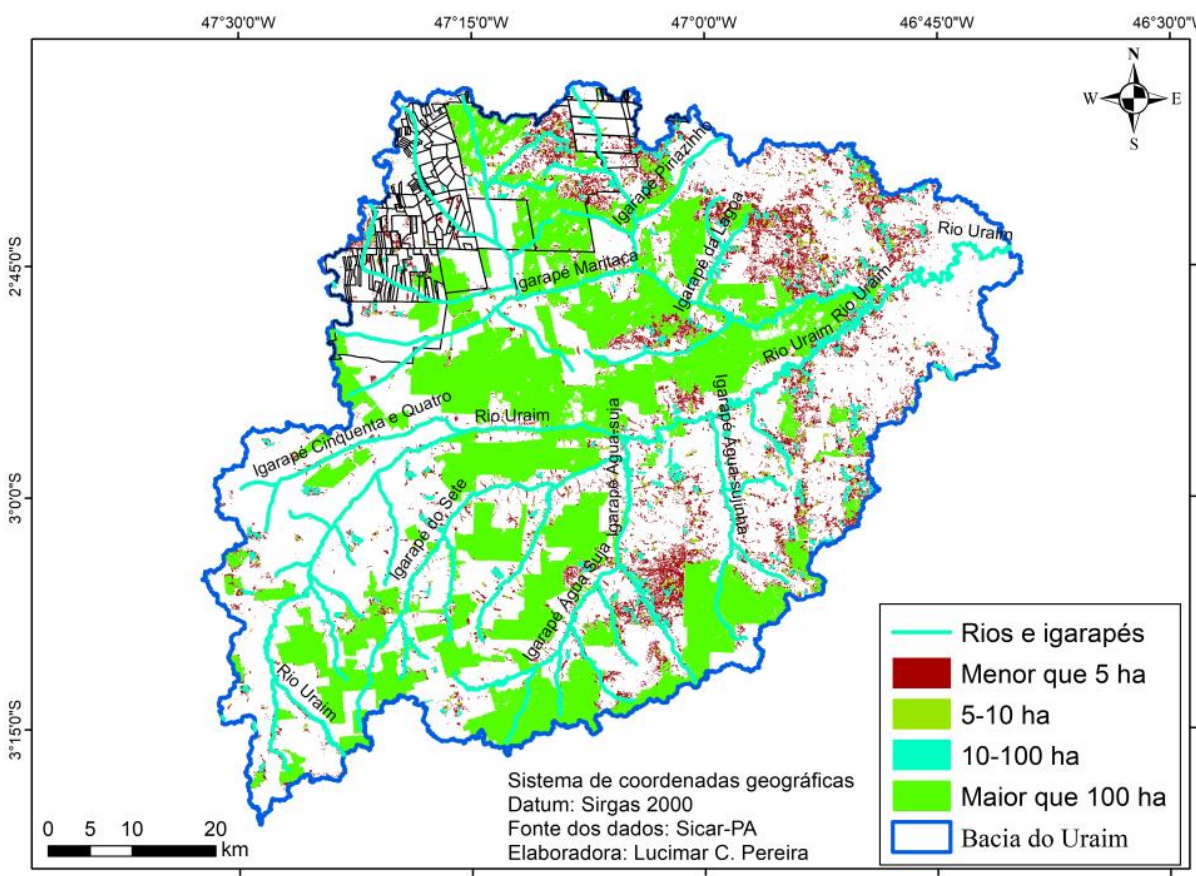

Organizadores: Autores (2019).

\section{Métricas de paisagem}

O produto da análise dos fragmentos florestais a partir de métricas de paisagem, para as classes de tamanho e para todos os fragmentos da bacia encontram-se na tabela 3.

Tabela 3 - Índices de ecologia da paisagem calculados para os fragmentos florestais no limite da bacia do rio Uraim, Paragominas-PA.

\begin{tabular}{|c|c|c|c|c|c|c|c|}
\hline & & & & & \\
\hline & & & \multicolumn{5}{|c|}{ Classes de Tamanho } \\
\hline Grupo & $\begin{array}{c}\text { Métrica } \\
\text { s }\end{array}$ & Unidade & $\begin{array}{c}\text { Muito } \\
\text { pequeno } \\
\text { (<5 ha) }\end{array}$ & $\begin{array}{l}\text { Pequeno } \\
\text { (5-10 ha) }\end{array}$ & $\begin{array}{c}\text { Médio } \\
\text { (10-100 } \\
\text { ha) }\end{array}$ & $\begin{array}{c}\text { Grande } \\
(>100 \\
\text { ha) }\end{array}$ & $\begin{array}{c}\text { Todos } \\
(0,1 \mathrm{a} \\
>100 \mathrm{ha})\end{array}$ \\
\hline Área & CA & $\begin{array}{l}\text { Hectares } \\
\text { (ha) }\end{array}$ & $10.177,3$ & 2242,19 & 7215,24 & 120485 & 140557 \\
\hline \multirow{3}{*}{$\begin{array}{l}\text { Densidad } \\
\text { e e } \\
\text { Tamanho }\end{array}$} & MPS & $\begin{array}{l}\text { Hectares } \\
\text { (ha) }\end{array}$ & 0,36 & 6,84 & 28,18 & 1853,61 & 4,76 \\
\hline & NUMP & $\underset{I}{\text { Adimensiona }}$ & 28016,00 & 328,00 & 256,00 & 65 & 29.504 \\
\hline & PSSD & $\begin{array}{l}\text { Hectares } \\
\text { (ha) }\end{array}$ & 0,60 & 1,48 & 18,97 & 5316,51 & 264,25 \\
\hline \multirow[t]{2}{*}{ Borda } & TE & Metros (m) & $\begin{array}{c}8368190,0 \\
0\end{array}$ & $\begin{array}{c}1095940,0 \\
0\end{array}$ & $\begin{array}{c}2781560,0 \\
0 \\
\end{array}$ & $\begin{array}{c}1434110 \\
0 \\
\end{array}$ & $\begin{array}{c}2685800 \\
0 \\
\end{array}$ \\
\hline & ED & $\mathrm{m} / \mathrm{ha}$ & 822,24 & 488,78 & 385,51 & 119,028 & 191,08 \\
\hline \multirow{2}{*}{ Forma } & MSI & $\begin{array}{c}\text { Adimensiona } \\
\mathrm{I}\end{array}$ & 1,40 & 3,60 & 5,74 & 12,28 & 1,49 \\
\hline & AWMSI & $\begin{array}{c}\text { Adimensiona } \\
\text { I }\end{array}$ & 2,02 & 3,65 & 6,38 & 35,42 & 30,91 \\
\hline
\end{tabular}

Organizadores: Autores (2019). 
No que se refere às métricas de densidade e tamanho, a análise dos dados indicou média de tamanho (MPS) para todos os fragmentos analisados de maneira conjunta, sem distinção de diferentes classes de tamanho, de 4,76 ha. O desvio padrão do tamanho da mancha (PSSD) encontrado foi de 264,25 ha. Tal fator indica a existência de fragmentos florestais com tamanhos muito acima e/ou muito abaixo do valor médio para todos os fragmentos, como foi verificado para as classes de tamanhos grande e muito pequeno, com MPS de 1.853,61 ha e 0,36 ha e PSSD de 5.316,51 ha e 0,60 ha, respectivamente.

A análise das métricas de borda indicou um total de bordas (TE) menor para a classe de fragmentos pequenos (1.095.940), enquanto a classe dos fragmentos grandes apresentou um número mais elevado (14.341.100). Os fragmentos muito pequenos apresentaram total de bordas $(8.368 .190)$ menor do que os fragmentos grandes. Apesar de tal fator, semelhante ao verificado por Juvanhol et al. (2011), a relação entre total de bordas e contribuição em área foi maior para os fragmentos muito pequenos do que os fragmentos grandes. $O$ total de bordas para os fragmentos médios foi significativamente menor (2.781.560) do que os valores encontrados para a classe muito pequeno e grande.

Quanto à densidade de borda (ED), os fragmentos muito pequenos e pequenos apresentaram os maiores valores, contabilizando $1.311,02 \mathrm{~m}$ de borda por hectare enquanto os fragmentos médios e grandes apresentaram densidade de borda de 385,51 m/ha e 119,028 m/ha, respectivamente.

Juvanhol et al. (2011) destacam que a distinção verificada na quantidade de bordas, quando a densidade é considerada, se deve, sobretudo, aos valores de área ocupados por cada classe de tamanho dos fragmentos. Nesse viés, a densidade de bordas é inversamente proporcional à área ocupada por cada classe. Os resultados encontrados indicam um efeito de borda mais reduzido nos fragmentos grandes, indicando de tal maneira, um grau de conservação mais elevado.

As métricas total de borda (TE) e densidade de borda (ED) representam a maneira como os fragmentos florestais sofrem influência, em função da área, pelo meio circundante (SANTOS et al., 2017).

A forma dos fragmentos é outro fator que influencia no grau de impacto do efeito de borda. Os valores de índice de forma (MSI) indicaram que os fragmentos menores apresentaram maior regularidade no formato, (MSI igual a 1,40 para os fragmentos muito pequenos e 3,60 para a classe dos pequenos), em relação aos fragmentos médios e grandes, os quais apresentaram MSI igual a 5,74 e 12,28, respectivamente. De tal maneira, constatou-se que a irregularidade dos formatos dos fragmentos aumentou de acordo com o tamanho dos mesmos.

Em pesquisa realizada por Hentz et al. (2015), na bacia hidrográfica do rio Pirapozinho-SP, os autores também observaram valores para MSI que indicaram maior regularidade para os fragmentos menores. Os autores constataram que quanto menor a classe de área, mais regular são os fragmentos, em valores médios. Neste caso, os fragmentos foram comparados a forma de um círculo, portanto os valores mais próximos à forma circular, indicam maior regularidade (HENTZ et al., 2015).

O índice de forma médio ponderado pela área (AWMSI) para as classes de tamanho dos fragmentos florestais se mostrou superior aos valores verificados para o índice de forma médio (MSI). Esse fato indica que os fragmentos de maior área possuem forma mais irregular que a média. Tal relação é existente pelo fato de que, para o cálculo do AWMSI, os fragmentos recebem pesos em função do tamanho dos mesmos. Apesar dos índices indicarem maior regularidade para os fragmentos pequenos, o tamanho e a forma do fragmento natural (que surge com a regeneração) ou não natural (área de floresta que foi fragmentada) podem estar intrinsecamente ligados à borda (MCGARIGAL e MARKS,1995; PIROVANI, 2010). 


\section{CONSIDERAÇÕES FINAIS}

A classificação de uso e cobertura da terra demonstrou que a área da bacia apresenta um quantitativo elevado de áreas de agricultura, capoeira, pastagem e solo exposto, apesar da classe de floresta primária deter de um maior percentual. A classe de floresta primária se encontra em estado de fragmentação, apresentando um número elevado de fragmentos florestais com área menor que 5 ha.

As métricas de paisagem demonstraram que apesar dos fragmentos maiores estarem em menor número, a área ocupada por estes é bem mais elevada. Os fragmentos maiores apresentaram menor efeito de borda em relação aos menores, entretanto, as métricas de forma indicaram irregularidade mais acentuada para os fragmentos com maior área.

Nesse contexto, vale ressaltar que o perfil de desenvolvimento de atividades agropecuárias na bacia do Uraim é o fator chave para a fragmentação florestal e a análise desses fragmentos contribui como auxílio na tomada de decisões para melhor gestão da unidade, considerando a necessidade de corredores ecológicos.

\section{AGRADECIMENTOS}

À Coordenação de Aperfeiçoamento de Pessoal de Nível Superior (CAPES), ao Programa de PósGraduação em Geografia da Universidade Federal do Pará - UFPA pelas oportunidades e apoio. Ao Museu Paraense Emílio Goeldi pelo auxílio no desenvolvimento da pesquisa.

\section{REFERÊNCIAS}

ALVES, W. S.; MARTINS, A.P.; AQUINO, D.S; MORAIS, W. A.; PEREIRA, M. A. B.; SALEH, B. B. Análise do uso da terra, da cobertura vegetal e da morfometria da bacia do Ribeirão Douradinho, no Sudoeste de Goiás, Brasil. Revista Brasileira de Geografia Física, Recife, v. 12, n. 3, p. 1093-1113, 2019. https://doi.org/10.26848/rbgf.v12.3.p1093-1113

BRASIL. Lei $n^{\circ}$ 9.433, de 8 de janeiro de 1997. Institui a Política Nacional de Recursos Hídricos e cria o Sistema Nacional de Gerenciamento de Recursos Hídricos. Disponível em:< http://www.planalto.gov.br/ccivil_03/LEIS/L9433.htm>. Acesso em: 23 out. 2019.

COHEN, J. A Coefficient of Agreement for Nominal Scales. Educational And Psychological $\begin{array}{lllllll}\text { Measurement, Durham, } & \text { v. 20, n. } 1, \quad \text { p. } 37-46,\end{array}$ https://doi.org/10.1177/001316446002000104

DNIT. Departamento Nacional de Infraestrutura de Transportes. Atlas e mapas. 2015. Disponível em:<http://www.dnit.gov.br/mapas-multimodais/shapefiles. Acesso em: 04 mar. 2020.

EMBRAPA. Empresa Brasileira de Pesquisa Agropecuária. Embrapa solos. 2019. Disponível em:<https://www.embrapa.br/solos>. Acesso em: 10 out. 2019.

FERNANDES, R. A. B. Discursos de sustentabilidade: o caso Paragominas. 94 f. 2011. Dissertação (Mestrado) - Curso de Ciência da Informação. Universidade Federal do Rio de Janeiro, Rio de Janeiro, 2011.

HENTZ, A. M. K.; CORTE, A. P. D.; DOUBRAWA, B.; SANQUETTA, C. R. Avaliação da fragmentação dos remanescentes florestais da bacia hidrográfica do rio iguaçu - PR, Brasil. Enciclopédia Biosfera, Goiânia, v. 11, n. 21, p. 2842-2858, 2015.

IBGE. Instituto Brasileiro de Geografia e Estatística. Bases e referenciais: bases cartográficas, malhas digitais. 2015. Disponível em:<https://mapas.ibge.gov.br/bases-e-referenciais/basescartograficas/malhas-digitais>. Acesso em: 04 mar. 2020. 
IMAZON. Instituto do Homem e Meio Ambiente da Amazônia. Planejamento do Uso do Solo do Município de Paragominas Utilizando Dados Econômicos e Ecológicos. Ed. 9. 2013. Disponível em:<https://imazon.org.br/author/imazon/>. Acesso em 11 nov. 2019.

JUVANHOL, R. S.; FIEDLER, N. C.; SANTOS, A. R.; PIROVANI, D. B.; LOUZADA, F. L. R. O.; DIAS, H. M.; TEBALDI, A.L.C. Análise Espacial de Fragmentos Florestais: Caso dos Parques Estaduais de Forno Grande e Pedra Azul, Estado do Espírito Santo. Floresta e Ambiente, Rio de Janeiro, v. 18. n. 4, p. 353-364, 2011. https://doi.org/10.4322/floram.2011.055

LANDIS, J.R. e KOCH, G.G. The measurement of observer agreement for categorical data. Biometrics, Porto, v.33, n.1, p. 159-174, 1977 . https://doi.org/10.2307/2529310 PMid:843571

MCGARIGAL, K.; MARKS, B. J. 1995. Fragstats: Spatial pattern analysis program for quantifying landscape structure. Reference manual. For. Sci. Dep. Oregon State University. Corvallis Oregon. 59 p. https://doi.org/10.2737/PNW-GTR-351

NASA. National Aeronautics and Space Administration. Earth Data 2011. Disponível em:< https://vertex.daac.asf.alaska.edu/>. Acesso em: 20 abr. 2019.

NASCIMENTO, T.; FERNANDES, L. Mapeamento do Uso e Ocupação do Solo em uma Pequena Bacia Hidrográfica da Amazônia. Ciência e Natura, Santa Maria, v. 39, n. 1, p. 170178, 2017. https://doi.org/10.5902/2179460X21737

PEREIRA, J.; OLIVEIRA, H.; BITENCOURT, E.; LOUREIRO, G. Geoprocessamento como ferramenta de caracterização morfométrica da bacia hidrográfica do rio Parauapebas, PA. Revista Geonorte, Manaus, v. 10, n. 34, p. 168-182, 2019. https://doi.org/10.21170/geonorte.2019.V.10.N.34.168.182

PEREIRA, L. C.; CORREAA, D. L.; SILVA, C. C. P.; LIMA, M. J. A.; SOUZA, R. R. N.; SILVA, A. C. S. Estudo das formas de ocupação das APPs de partes do rio Uraim Paragominas-PA. Blucher Proceedings, online, v. 3, n. 2, p. 408-415, 2016. https://doi.org/10.5151/engpro-eneeamb2016-rh-018-4900

PIROVANI, D. B. Estudo da fragmentação florestal, dinâmica e ecologia da paisagem na bacia hidrográfica do rio Itapemirim, ES. Dissertação (Mestrado em Ciências Florestais) - Jerônimo Monteiro: Universidade Federal do Espirito Santo. 2010.

REIS, J. C.; ALVES, J. S. Impactos ambientais decorrentes do uso e ocupação do solo na microbacia do córrego água quente em Rio Quente, Goiás. Revista Eletrônica Geoaraguaia, Cuiabá, v. 7, n. 1, p. 22 - 43, 2017.

REX, F. E.; CORTE, A. P. D.; KAZAMA, V. S.; SANQUETTA, C. R. Análise métrica da cobertura florestal da bacia hidrográfica do rio Pequeno - PR. Biofix Scientific Journal, Curitiba, v. 3, n. 1, p. 184-192, 2018. https://doi.org/10.5380/biofix.v3i1.58382

RIBOLDI, L. C. O.; RÉCIO, L. V.; FERREIRA, I. J. M.; FERREIRA, H. D.; COUTO, E. V. Análise das métricas de ecologia de paisagem em fragmentos florestais no município de salgado Filho/PR. Revista Geomae, Campo Mourão, v. 8, n. 3, p. 177-185, 2017.

RODRIGUES, B. M.; OSCO, L. P.; ANTUNES, P. A.; RAMOS, A.P. M. Avaliação da influência do uso e cobertura da terra na qualidade das águas superficiais da bacia hidrográfica do rio Pirapozinho (SP). Revista Brasileira de Geografia Física, Recife, v. 12, n. 3, p. 738-753, 2019. https://doi.org/10.26848/rbgf.v12.3.p738-753 
SANTOS, I.; SALIM, S.; PEREIRA, P. Caracterização do reflorestamento de Paricá na microrregião de Paragominas-PA. Revista Agroecossistemas, Belém, v. 10, n. 1, p. 145-158, 2018. https://doi.org/10.18542/ragros.v10i1.5109

SANTOS, J. F. C.; MENDONÇA, B. A. F.; ARAÚJO, E. J. G.; ANDRADE, C. F. Fragmentação florestal na Mata Atlântica: o caso do município de Paraíba do Sul, RJ, Brasil. Revista Brasileira de Biociências, Porto Alegre, v. 15, n. 3, p. 151-158, 2017.

SAUNDERS, D. A.; HOBBS, R. J.; MARGULES, C. R. Biological Consequences of Ecosystem Fragmentation: A Review. Conservation Biology, New York, v. 5, n. 1, p. 18-32, 1991. https://doi.org/10.1111/j.1523-1739.1991.tb00384.x

SICAR. Sistema Nacional de Cadastro Ambiental Rural. Imóveis rurais 2019. Disponível em:< http://www.car.gov.br/publico/municipios/downloads.>. Acesso em: 10 nov. 2019.

TURNER, I.M. Species loss in fragments of tropical rain forest: a review of the evidence. Journal of Applied Ecology, Londres, v.33, n.2 p.200-209, 1996. https://doi.org/10.2307/2404743

USGS. United States Geological Survey. Imagem OLI Landsat 8 órbita/ponto 222/62 de 02/07/2018. Disponível em: https://earthexplorer.usgs.gov/. Acesso em: 20 nov. 2019.

VIANA, V. M.; PINHEIRO, L. A. F. V. Conservação da biodiversidade em fragmentos florestais. Série Técnica IPEF, Piracicaba-SP, v. 12, n. 32, p. 25-42, dez. 1998.

Recebido em: 13/11/2019

Aceito para publicação em: 22/04/2020 